\title{
eJRIEPS
}

Ejournal de la recherche sur l'intervention en éducation physique et sport

$38 \mid 2016$

Varia

\section{El « modelo integrado técnico-táctico » de enseñanza deportiva. Origen, contextualización y características metodológicas}

Víctor López-Ros

\section{OpenEdition}

Journals

Edición electrónica

URL: http://journals.openedition.org/ejrieps/892

DOI: 10.4000/ejrieps.892

ISSN: 2105-0821

Editor

ELLIADD

Referencia electrónica

Víctor López-Ros, «El « modelo integrado técnico-táctico » de enseñanza deportiva. Origen, contextualización y características metodológicas », eJRIEPS [En ligne], 38 | 2016, mis en ligne le 21 juin 2019, consulté le 01 août 2019. URL : http://journals.openedition.org/ejrieps/892 ; DOI : 10.4000/ ejrieps.892

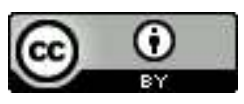

La revue eJRIEPS est mise à disposition selon les termes de la Creative Commons Attribution 4.0 International License. 


\section{eJRIEPS 38 avril 2016}

El « modelo integrado técnico-táctico » de enseñanza deportiva.

Origen, contextualización y características metodológicas.

Víctor López-Ros

Cátedra de Deporte y Educación Física-Centro de Estudios Olímpicos, Universidad de Girona, Catalunya, España.

\section{Resumen}

El interés por mejorar la enseñanza deportiva, y en especial los deportes con contenidos técnicos y tácticos, hizo que durante la última parte del siglo XX aparecieran diferentes modelos de enseñanza. Uno de los modelos que apareció en España fue el Modelo Integrado Técnico-Táctico. En el presente artículo, tomando como referencia las publicaciones iniciales, explicamos el origen, las influencias recibidas y el contexto en el que se diseñó y se desarrolló dicho modelo. Mostramos sus características fundamentales y ponemos énfasis en distintos aspectos metodológicos subyacentes relacionados con la intervención docente. Tiene especial interés ubicar adecuadamente el modelo en una perspectiva socio-constructivista y con la voluntad de favorecer un aprendizaje significativo. Asimismo, situamos el Modelo Integrado Técnico-Táctico en relación con otros modelos, sus particularidades y algunos avances actuales. Finalmente mostramos aspectos sobre los que hay que profundizar para que sea una herramienta útil en la enseñanza comprensiva del deporte.

Palabras clave : enseñanza de los deportes, modelo integrado, técnica-táctica, aprendizaje significativo, perspectiva constructivista, comprensión.

\section{Introducción}

La enseñanza de los deportes ha experimentado importantes cambios a lo largo de los últimos años. Sobre todo por lo que se refiere a los deportes con contenidos técnicos y tácticos, y muy especialmente en los comúnmente llamados deportes colectivos. Durante el final del siglo XX e inicios del siglo XXI asistimos a una eclosión de modelos de enseñanza, determinada en gran medida por la necesidad de un revulsivo frente a los modelos pedagógicos tradicionales de corte técnico y mecanicista. El modelo con mayor impacto en el ámbito académico es el Teaching Games for Understanding (TGfU) (Bunker 


\section{eJRIEPS 38 avril 2016}

\& Thorpe, 1992) inglés, probablemente debido al dominio de las revistas de habla inglesa y a su ámbito de influencia. Sin embargo, en otros países también se aprecia esta necesidad de cambio y se proponen modelos alternativos que no emanan exclusivamente de la influencia del TGfU, sino que en parte dependen de otras tradiciones pedagógicas, como por ejemplo distintas propuestas francesas (Bouthier, 1984, 2014 ; Gréhaigne, Wallian \& Godbout, 2005). Cierto es, a nuestro entender, que la aparición de todos estos modelos comparte la necesidad de un cambio en la metodología de la enseñanza que surge fundamentalmente de las limitaciones apreciadas en el quehacer diario de maestros, profesores y entrenadores en el uso de perspectivas de enseñanza más tradicionales.

El objetivo del presente artículo es mostrar el origen y el contexto en el que aparece el Modelo Integrado Técnico-Táctico (MIT-T) (Castejón \& López Ros, 1997 ; López Ros \& Castejón, 1998a, 1998b, 2005) en España, así como las características metodológicas fundamentales relacionadas con propuestas planteadas en otros modelos o desde otras perspectivas. Nos interesa, más allá de mostrar los rasgos distintivos del MIT-T, evidenciar los motivos y planteamientos subyacentes que ubican el modelo en la perspectiva constructivista y su posible utilidad en la enseñanza. En España, como en otros países, aparecen distintos modelos de enseñanza que comparten elementos y difieren en otros, de forma que en algunos casos son difíciles de definir con absoluta nitidez (Castejón, Giménez Fuentes-Guerra, Jiménez Jiménez, López-Ros, 2003). Sin embargo, resulta importante considerar que el objetivo compartido por estos modelos se encuentra más en su utilidad para la práctica docente que en su pertinencia científica y académica. Si bien las evidencias empíricas y científicas son fundamentales, en última instancia el valor y efecto de los modelos se halla en su uso en los centros de enseñanza y de entrenamiento. Esta cuestión es común a distintos modelos. Baste señalar que uno de los aspectos todavía pendientes de mejora en el caso del TGfU es que, a pesar del apoyo académico recibido, ha tenido muy poco impacto en los centros de enseñanza (Stolz \& Pill, 2014; Sánchez-Gómez, Devís-Devís \& Navarro-Adelantado, 2014), entre otras cosas porque los docentes tienen fuertes dificultades para su uso (Díaz-Cueto, Hernández-Álvarez \& Castejón, 2010).

El diseño y desarrollo del MIT-T no tiene otra pretensión que intentar ser una herramienta útil para los enseñantes. Como cualquier recurso pedagógico, no debería tomarse en consideración al margen del contexto teórico en el que se halla inmerso ni de las 


\section{eJRIEPS 38 avril 2016}

intenciones educativas perseguidas. El MIT-T busca favorecer un aprendizaje contextualizado, comprensivo y lo más significativo posible.

\section{Origen y antecedentes del modelo}

El MIT-T aparece en 1998 en dos artículos en la "Revista de Educación Física. Renovación de la teoría y de la práctica” (López Ros \& Castejón, 1998a, b), aunque el esquema básico del modelo ya había sido publicado previamente en 1997 en un capítulo en el libro "Manual del Maestro Especialista en Educación Física” (Castejón \& López Ros, 1997). Estos dos artículos iniciales mostraban los principales aspectos teóricos y prácticos con el objetivo de que los maestros y entrenadores pudieran emplearlo en su quehacer diario. En este sentido, no se diseñó ni se presentó un modelo complejo, sino que se pensó en una estructura simplificada con el objetivo de que fuera útil y funcional para aquellos que lo emplearan en la enseñanza deportiva. Debido a que las primeras interpretaciones del modelo mostraban algunas confusiones y dificultades, en 2005 se publicó un artículo que explicaba con mayor profusión los aspectos esenciales del mismo (López Ros \& Castejón, 2005).

Como hemos indicado brevemente, el MIT-T se inscribe en una perspectiva de enseñanza que busca generar un aprendizaje funcional, contextualizado, comprensivo y significativo de las acciones deportivas. Ello implica, entre otros aspectos, trabajar conjunta e integradamente las dimensiones técnica y táctica de las acciones deportivas. Es decir, aprender la dimensión técnica en situaciones tácticas y la táctica en condiciones de exigencia técnica. Asimismo, pretende que los jugadores aprendan comprendiendo el sentido de sus acciones. No es solo una cuestión de que los jugadores aprendan a hacer, o de que emerjan las respuestas supuestamente idóneas producto de la autoorganización del sistema y como consecuencia de ajustar las condiciones de realización de las actividades. El modelo busca que los deportistas construyan un conocimiento lo más significativo posible y entiendan el por qué y el para qué de lo que hacen.

EI MIT-T se incluye en la orientación conocida como Enseñanza Comprensiva del Deporte (ECD) (Castejón, 2003, 2010), y comparte muchos aspectos con distintos modelos agrupados bajo la perspectiva del Games Centred-Approach (Harvey \& Jarrett, 2013; Oslin \& Mitchell, 2006). La ECD recoge diferentes ideas y propuestas que aparecen en la década de los 80 y 90 del pasado siglo como reacción a una perspectiva "tecnicista" y "mecanicista" de la enseñanza deportiva que imperaba hasta el momento (p.e. Castejón, 1995; Devís, 1990). Dicha perspectiva preconizaba que los deportistas aprendieran en 


\section{eJRIEPS 38 avril 2016}

primer lugar, y de forma mecanizada, los aspectos técnicos básicos de las distintas acciones del deporte correspondiente, basándose fundamentalmente en el criterio de eficiencia del gesto y de descomposición de las acciones objeto de aprendizaje. Este aprendizaje técnico era considerado, desde el punto de vista de la secuenciación, un primer paso necesario para un posterior aprendizaje táctico simplificado, que a su vez era un paso imprescindible y necesario para el aprendizaje más complejo en situaciones de juego. Por contraposición y como reacción a estos planteamientos aparecieron en España diferentes modelos y alternativas que sugerían en la mayoría de los casos empezar por la táctica o por situaciones globales (p.e. Blázquez, 1986; Devís, 1990; Lasierra \& Lavega, 1997).

2. 1. Antecedentes e influencias del modelo.

Una parte de las ideas subyacentes al MIT-T se encuentran en los modelos franceses de enseñanza de los deportes colectivos. Estos modelos se agrupan en la llamada "Pedagogía de los Modelos de Decisión Táctica" (PMDT) (Bouthier, 1984, 2014; Gréhaigne, Wallian \& Godbout, 2005), entendida como una perspectiva que propugna la enseñanza centrada en la táctica, y en favorecer la comprensión por parte de los jugadores durante el proceso de aprendizaje. La "aproximación táctica" francesa (Bouthier, 1984) basada, entre otros, en las ideas y propuestas de Teodorescu (1977) y de Deleplace (1979) tuvo un impacto considerable en zonas de influencia francesa y también en áreas colindantes, entre ellas Cataluña. Asimismo cabe destacar la influencia de los trabajos de Bayer (1986, 1987), basados sobre todo en el estructuralismo y el funcionalismo, y la perspectiva proveniente de la enseñanza del balonmano. En el momento del desarrollo del MIT-T los modelos didácticos y las propuestas pedagógicas que trabajaban con la "pedagogía de la situaciones" y con la resolución de problemas (Bonnet, 1988; Famose, 1991) representaron una alternativa que tuvo influencia en las nuevas orientaciones metodológicas. Autores españoles, como por ejemplo Blázquez (1986), desarrollaron propuestas en las que se aprecia claramente esta influencia de orientación francesa.

Por otro lado, cabe destacar el trabajo de F. Mahlo (1974), especialmente por lo que se refiere a la noción de "acto táctico". Este trabajo representó una ruptura epistemológica en la explicación de la acción deportiva en contextos tácticos. Especialmente por el hecho de señalar que no era pertinente considerar la acción de un jugador sin atender al contexto colectivo (del juego), de la misma forma que había que considerar la "ejecución motriz" como una parte de la acción táctica y, por tanto, la dimensión técnica debía entenderse en 


\section{eJRIEPS 38 avril 2016}

el contexto táctico. En coherencia con ello, Mahlo (1974) señalaba distintos momentos dentro del acto táctico según cuál fuera el principal mecanismo implicado (percepción, decisión, ejecución). Dichos mecanismos estaban relacionados entre sí pero al mismo tiempo tenían funciones y características diferenciadas. Esta idea estaba fuertemente impregnada por el Procesamiento de la Información como marco teórico explicativo de la cognición humana. La influencia de Mahlo se aprecia también en los modelos franceses, y así lo señalan distintos trabajos (Bouthier, 2014; Gréhaigne, Richard \& Griffin, 2005; Gréhaigne, Wallian \& Godbout, 2005).

Debemos indicar también la incidencia en el MIT-T del Teaching Games for Understanding (TGfU) de Bunker y Thorpe (1982). El TGfU llega a España de la mano de diferentes trabajos, entre ellos, los del ex-alumno de Loughborough José Devís (Devís, 1992; Devís \& Peiró, 1992). Su impacto en España está en la línea del recibido en otros países, especialmente en los del ámbito anglosajón en los que aparecen modelos y propuestas como el Game Sense, el Play Practice o el Tactical Games Approach (Sánchez-Gómez, Devís-Devís \& Navarro-Adelantado, 2014). Dado el enorme empuje recibido en las dos últimas décadas, existen infinidad de trabajos referidos tanto al desarrollo conceptual del modelo (p.e. Kirk \& McPhail, 2002), como a distintos aspectos de análisis y aplicación de los principios pedagógicos (p.e. Holt, Strean \& García Bengoechea, 2002), como de revisión del estado actual, (p.e. Stolz \& Pill, 2014), y también acerca de la compatibilidad o no con otras perspectivas de enseñanza del deporte (p.e. Clemente, 2012; Tan, Chow \& Davids, 2012). En su momento, la importancia del mismo estuvo relacionada con el hecho de plantear una enseñanza que centraba su atención en el aprendiz y en la participación activa del mismo en el proceso de aprendizaje. Ello hizo que se vinculara este modelo a una perspectiva constructivista del aprendizaje (p.e. Gréhaigne, Godbout \& Bouthier, 1999; Griffin \& Patton, 2005). El TGfU preconiza empezar jugando y supedita los comportamientos de los jugadores a su eficacia y funcionalidad en las situaciones de juego. Es decir, la decisión táctica y la habilidad técnica solo tienen relevancia en la medida en que son útiles en las situaciones de juego. Según este modelo, es a partir de las situaciones de juego que los aprendices desarrollan la conciencia táctica en la que se enmarca la toma de decisiones. Estas decisiones establecen qué habilidad emplear y en qué condiciones, y posteriormente se lleva a cabo una acción ajustada a las demandas de la situación. Si bien existen múltiples consideraciones al respecto (p.e. Griffin \& Butler, 2005; Griffin, Brooker \& Patton, 2005; Stolz \& Pill, 2014), este modelo representó un cambio sustancial en la forma de 


\section{eJRIEPS 38 avril 2016}

considerar la enseñanza deportiva en el ámbito anglosajón. En términos generales es correcto señalar que el MIT-T presenta rasgos comunes con el TGfU y también con la PMDT, sin embargo los distintos modelos muestran también características que les son propias (López-Ros, Castejón, Bouthier \& Llobet, 2015).

Además de las influencias mencionadas, el MIT-T se desarrolla en un momento de fuerte debate acerca de si era preferible empezar la iniciación a los deportes técnico-tácticos por la técnica o por la táctica. Distintos trabajos, entre ellos los publicados en el monográfico del Journal of Teaching in Physical Education editado por J. Rink (Rink, 1996), o el trabajo de Turner y Martinek (1992) señalaban que no existían evidencias claras de que fuera mejor empezar priorizando la técnica o la táctica; del mismo modo que mostraban las dificultades de comparar los trabajos e investigaciones realizadas hasta el momento. En este contexto se llevaron a cabo distintas investigaciones también en España, que intentaban dar respuesta a la pregunta de si era mejor empezar por la técnica, por la táctica, o por ambas simultáneamente (p.e. Castejón et al., 2002; García Herrero \& Ruiz Pérez, 2003).

Todo ello dibujaba un escenario de cambio y de necesidad de búsqueda de nuevas formas de enseñanza de los llamados "deportes colectivos". Por un lado, la enseñanza basada en la primacía de la técnica sobre la táctica, y en el uso de metodologías analíticas resultaba insuficiente para garantizar un aprendizaje adecuado durante las etapas de iniciación. Y por otro lado, aparte de los trabajos franceses sobre la PMDT, no se disponía de evidencias suficientemente concluyentes acerca de empezar la enseñanza por la táctica y supeditar en cualquier circunstancia la enseñanza de la técnica a la táctica.

2. 2. ¿Bajo qué perspectiva teórica de la enseñanza y el aprendizaje?

De acuerdo con lo señalado por Rink (2001), todos los métodos y modelos de enseñanza se basan, en el fondo, en una forma particular de considerar el aprendizaje. EI MIT-T reposa sobre una perspectiva constructivista del aprendizaje que, al mismo tiempo, define aspectos fundamentales sobre la forma de enseñar. El uso de la perspectiva constructivista como marco de referencia es compartido por distintos modelos, si bien no parece existir una unanimidad acerca de qué significa y conlleva dicha perspectiva constructivista. Por lo que respecta al MIT-T, y de acuerdo con los trabajos que señalan que no existe "el constructivismo" como una teoría única y unitaria (Cubero, 2005; Rovegno \& Dolly, 2006), resulta necesario explicar qué perspectiva u orientación constructivista subyace a dicho modelo. Algunos de los aspectos que aquí señalaremos 


\section{eJRIEPS 38 avril 2016}

en relación a la perspectiva constructivista pueden verse con mayor detalle en López Ros (2010).

El modelo busca en su origen favorecer que los deportistas lleven a cabo un aprendizaje lo más significativo posible. Uno de los ejes sobre los que se soporta este propósito es el desarrollo de la funcionalidad y utilidad de las acciones técnicas y tácticas empleadas. En el momento de desarrollar el modelo partíamos de la idea de que el aprendizaje es más significativo en la medida en que el aprendiz le puede otorgar sentido a aquello que aprende. $Y$ esto es más posible que suceda si lo que se aprende es útil y funcional para resolver una determinada situación, y si ello se apoya en la comprensión de las propias acciones y de los propios aprendizajes. El modelo propone emplear "situacionesproblema" de forma que la acción (más técnica o más táctica) objeto de aprendizaje sea la opción fundamental y deseable por parte del jugador para resolver el problema planteado. Ello facilita que la acción tenga significación (y el aprendiz pueda otorgarle sentido) puesto que es útil y funcional para resolver un problema motriz determinado, y abre la puerta a la comprensión. El desarrollo de la comprensión y el acceso al significado no emanan directamente de la participación en "situaciones problema", pero el uso de la acción en el contexto ayuda a que el aprendiz le pueda otorgar sentido. Esta idea del aprendizaje significativo es deudora de los trabajos de Ausubel (pueden verse versiones revisadas, por ejemplo, en Ausubel, 2000; y en Ausubel, Novak \& Hanesian, 1990), y de aportaciones de otros autores acerca de las condiciones para favorecer un aprendizaje lo más significativo posible en entornos educativos (p.e. Coll, 1988).

De igual forma, los supuestos de la perspectiva constructivista sociocultural y las ideas de Vigotsky (1979) tuvieron una fuerte influencia. Por un lado, el concepto de "Zona de Desarrollo Próximo" (ZDP) y los procesos de ayuda pedagógica para favorecer el aprendizaje. Y por otro lado, el supuesto de que la mente está mediada semióticamente y que, por lo tanto, los recursos semióticos son fundamentales para favorecer el desarrollo de los procesos psicológicos superiores. Ambas ideas han sido ampliamente estudiadas y analizadas en las últimas décadas en el ámbito educativo (p.e. Daniels, 2001). Si bien en el momento de elaborar el modelo el alcance de las ideas vigotskianas en España era menor que en la actualidad, los trabajos en el ámbito de la psicología aparecidos entonces ya ponían un gran énfasis en la mediación semiótica de la mente y también en el concepto de ZDP (p.e. Rivière, 1988; Vila, 1987). En el caso de la enseñanza deportiva su incidencia era en aquel momento minúscula, y solo algunas propuestas se desarrollaron desde esta perspectiva (p.e. Castejón \& López Ros, 1997). 


\section{eJRIEPS 38 avril 2016}

El concepto de ZDP tiene múltiples consecuencias. Una de fundamental es que entiende que el aprendizaje sólo es posible cuando la dificultad de la tarea propuesta se sitúa por encima del nivel de desarrollo real de los aprendices, es decir, representa un problema por resolver de grado superior al nivel inicial de los sujetos. Asimismo, el nivel de dificultad no puede ser excesivo puesto que de lo contrario sería irresoluble para un nivel determinado. La posibilidad de resolver un problema de un nivel superior al real, y de aprender sobre el mismo, se da gracias a la ayuda de alguien más experto con el cual el aprendiz interacciona continuamente. La actividad del aprendiz y del enseñante debe entenderse como una actividad conjunta y compartida, en la cual el enseñante ofrece la ayuda pedagógica pertinente en cada momento, hasta que el aprendiz es capaz de resolver por sí solo la tarea en cuestión. El enseñante ayuda y progresivamente va retirando dicha ayuda. Este proceso fue conceptualizado por Wood, Bruner \& Ross (1976) como la metáfora del andamiaje (scaffolding) y en los últimos años ha generado una cantidad considerable de trabajos (puede verse una revisión del impacto en el ámbito educativo en Van de Pol, Volman \& Beishuizen, 2010). Algunos de ellos han mostrado el carácter dinámico del proceso, mientras que otros han buscado aspectos distintivos del proceso de interacción, señalando como un hecho destacado que la ayuda sea "contingente" (Van de Pol, Volman \& Beishuizen, 2010, 2011). El concepto de scaffolding también se ha empleado en el estudio y análisis de la enseñanza deportiva (Chen, Rovegno, Cone \& Cone, 2012; López Ros, 2003; Wright, McNeill \& Fry, 2009).

Para que la ayuda y la influencia educativa se pueda llevar a cabo, es necesario el proceso de comunicación y de negociación entre el enseñante y el aprendiz a partir de establecer una red de significados compartidos. Así, el tipo de recursos empleados en los procesos interactivos y, especialmente para facilitar la resolución de la tarea y para favorecer la construcción de conocimiento son especialmente relevantes (Coll, Onrubia \& Mauri, 2008). En el momento de la aparición del MIT-T, el análisis y estudio de los procesos interactivos estaba aún bajo el influjo de paradigmas fuertemente vinculados a la perspectiva del proceso-producto, y empezaban a tomar fuerza planteamientos de corte ecológico y constructivista. La perspectiva asumida por el MIT-T entendía la interacción como la organización de la actividad conjunta entre enseñante y aprendices, de forma que los recursos semióticos empleados solo pueden considerarse en el marco de dicha organización conjunta, y por tanto en el marco de las intenciones, reglas y formatos de organización social definidos en un momento determinado. Asimismo, se consideraba que la posibilidad real de favorecer un aprendizaje comprensivo reposaba no solo en el diseño 


\section{eJRIEPS 38 avril 2016}

de tareas adecuadas, sino también en el uso de dichos recursos semióticos. Para ello se indicaba la pertinencia y necesidad de que el enseñante y los aprendices compartieran una red de significados en relación a la situación educativa creada (López Ros \& Castejón, 1998a).

De acuerdo con lo señalado, el MIT-T considera que las tareas de enseñanza deben ser pensadas como un problema a resolver por parte del aprendiz, siendo la adecuada complejidad del problema un elemento fundamental. Es decir, (a) el problema debe ser resoluble, pero suficientemente exigente para que se requiera de ayuda; (b) la clave del éxito en la resolución de la tarea debe estar en el contenido objeto de aprendizaje, sin que haya interferencias de otros aspectos de la acción deportiva que impidan su resolución; y (c) la ayuda debe entenderse como el uso ajustado de los recursos semióticos que permiten avanzar hacia la autonomía en la resolución del problema y en la comprensión de los aspectos que lo hacen posible. En este sentido, el MIT-T se entiende como un modelo de enseñanza comprensiva del deporte.

\section{Estructura y características del modelo}

3. 1. Una visión global

Como ya se ha indicado, el modelo se diseñó para que fuera sencillo, funcional y adaptable y, en este sentido "tiene que contener los elementos explicativos mínimos que permitan entender su dinámica y, al mismo tiempo, tiene que ser una simplificación de la realidad. Se trata de mostrar las directrices básicas que puedan servir de guía para una aplicación didáctica” (López Ros \& Castejón, 2005: 41). El enseñante y el entrenador deben encontrar las ideas fundamentales para poder emplear el modelo de forma sencilla y, al mismo tiempo, se tiene que poder adaptar a las particularidades de cada situación de enseñanza. Esta adaptabilidad es importante y debe basarse en ideas genéricas y globales que permitan su uso en la enseñanza de distintos deportes y en diferentes contextos.

EI MIT-T propone empezar el proceso enseñando el dominio técnico y el dominio táctico complementariamente y en paralelo, de forma que los deportistas deben aprender los aspectos tácticos y técnicos correspondientes a un nivel determinado al mismo tiempo y de forma contextualizada. Estos aprendizajes deben corresponderse con las situaciones de juego colectivo de referencia que son, al fin y al cabo, los escenarios en los que las acciones tácticas y técnicas tienen sentido y donde adquieren su significación. Esta idea del aprendizaje contextualizado de las acciones en relación al entorno más próximo y 


\section{eJRIEPS 38 avril 2016}

también al marco de referencia del juego colectivo aparece recogida en múltiples trabajos (p.e., Gréhaigne, Godbout \& Bouthier, 1999; Gréhaigne, Richard \& Griffin, 2005) y es coherente también con las ideas del TGfU y de la PMDT francesa (Bouthier, 2014; Gréhaigne, Wallian \& Godbout, 2005). Asimismo, existen puntos de coincidencia con una parte de los conceptos subyacentes al "Situated learning" (Rovegno, 2006) y con la "Constraints-led approach" (Renshaw, Chow, Davids \& Hammond, 2010), especialmente en los aspectos que señalan la interdependencia de la acción con la situación específica en la que tiene lugar. Más allá de coincidencias y discrepancias entre las distintas perspectivas, lo importante es el interés por el aprendizaje de la acción deportiva en el contexto en el que ésta se lleva a cabo en condiciones normales.

Finalmente el modelo, al igual que Deleplace (1979) y Bouthier (2014), propone realizar una progresión de los aprendizajes en espiral, de forma que el aprendiz hace frente muchas veces a tareas conocidas aunque en cada etapa (o vuelta de la espiral) la tarea en cuestión tiene un mayor nivel de complejidad. Es decir, la actividad mantiene los aspectos estructurales básicos pero incorpora variaciones que generan un aumento de la complejidad. La progresión en espiral se emplea tanto en las situaciones más sencillas de aprendizajes más individuales o con estructuras colectivas sencillas (p.e. 2 vs 2), como en las situaciones de juego colectivo de referencia. Esta idea de aprendizaje circular que evoluciona como una espiral aumentando el nivel de complejidad a partir de tomar en consideración los conocimientos previos ya aparecía parcialmente en el TGfU, influenciado por las ideas de Bruner acerca del currículum en espiral (Sánchez-Gómez, Devís-Devís \& Navarro-Adelantado, 2014), y en otras propuestas de enseñanza (p.e. Blázquez, 1986).

En la figura que sigue se puede ver el modelo original,

1.- Dominio de las habilidades y destrezas básicas
$\begin{aligned} & \text { 2.- Enseñanza de la táctica con } \\
& \text { implicación de pocos elementos }\end{aligned}$
técnicos \begin{tabular}{l}
$\begin{array}{l}2 .- \text { Enseñanza de la técnica con } \\
\text { implicación de pocos elementos } \\
\text { tácticos }\end{array}$ \\
\hline 3.- Presentación de situaciones de juego similares al deporte definitivo con \\
aplicación de los elementos técnicos y tácticos aprendidos \\
\hline $\begin{array}{l}\text { 4.- Enseñanza de la táctica con } \\
\text { implicación de pocos elementos }\end{array}$ \\
$\begin{array}{l}\text { 4.- Enseñanza de la técnica con } \\
\text { implicación de pocos elementos } \\
\text { tácticos }\end{array}$ \\
\hline $\begin{array}{l}\text { 5.- Presentación de situaciones de juego similares al deporte definitivo con } \\
\text { aplicación de los elementos técnicos y tácticos aprendidos }\end{array}$
\end{tabular}

Figura 1. Esquema del "Modelo integrado técnico-táctico" de enseñanza de los deportes colectivos (Castejón \& López Ros, 1997; López Ros \& Castejón, 1998a, 2005). 


\section{eJRIEPS 38 avril 2016}

\section{2. Caracterización y estructura fundamental.}

Tal como aparece en la Figura 1, el modelo sugiere empezar a partir de un aspecto básico y fundamental:

1.- Dominio de las habilidades motrices básicas en el nivel de eficacia y de disponibilidad variable.

El desarrollo y dominio de lo que podríamos llamar motricidad básica (habilidades motrices básicas) es una condición indispensable para garantizar un aprendizaje técnico y táctico adecuado. Sin embargo, hay múltiples cuestiones en relación a este apartado que son importantes y que exceden el objeto de este artículo. De forma muy simplificada, hay que considerar, por un lado, las particularidades evolutivas en la aparición de las distintas habilidades motrices básicas. Es decir, dichas habilidades aparecen de forma progresiva a lo largo de la infancia de manera que unas se aprenden antes que otras (los niños y niñas aprenden antes a andar que a correr o a saltar). Ello comporta una diferencia importante en la cantidad de tiempo de experiencia de una habilidad derivado simplemente del hecho del momento de su aparición. Asimismo, una vez adquirida una habilidad motriz básica, el uso que de ella se hace es muy diverso. Dado que es bien conocido que el dominio de las habilidades motrices depende en parte de la cantidad y de la calidad de la práctica realizada, además del momento evolutivo de aparición, ello supone que los deportistas noveles presenten diferentes niveles de dominio en las distintas habilidades motrices básicas en función de la cantidad y calidad de uso de las mismas. Puesto que las habilidades deportivas son habilidades específicas 0 especializadas, esto tiene consecuencias y señala que el nivel de dificultad de los deportistas noveles en el aprendizaje de aspectos técnicos y tácticos puede ser muy variado en función de su grado de dominio de las habilidades motrices básicas implicadas en dicho deporte. Ello debe tenerse en cuenta antes de pasar al nivel 2 mostrado en la Figura 1, así como si debe hacerse mayor incidencia en el aspecto técnico o táctico en el inicio de esta fase 2.

En términos generales, y sin ánimo de extendernos, es importante que los aprendices dispongan de un bagaje de respuestas suficientemente amplio que facilite la adaptación de la acción a contextos variados. Desde nuestro punto de vista, el trabajo sobre la motricidad básica previa a la enseñanza deportiva específica debería entenderse como el desarrollo de la "competencia motriz" (Ruiz Pérez, 1995, 2001). Si bien estos aspectos han sido profusamente tratados por distintas teorías del aprendizaje motor, lo que aquí 


\section{eJRIEPS 38 avril 2016}

nos interesa señalar es que el dominio y adaptabilidad de la motricidad básica es un elemento importante que condiciona posteriores aprendizajes.

2.- Aprendizaje simultáneo de las dimensiones técnica y táctica de la acción deportiva.

El modelo parte de la idea de que las llamadas técnicas y tácticas deportivas son dos dimensiones necesarias de la acción de los jugadores en los deportes colectivos, de forma que preconiza un aprendizaje integrado de los dos dominios, el táctico y el técnico. Para que esto sea posible el modelo sugiere trabajar sobre los dos aspectos de forma conjunta, pero focalizando la atención del aprendizaje sobre uno de ellos. Cuando se trabaja sobre la dimensión táctica, se emplean los aspectos técnicos pero la dificultad principal de la actividad se encuentra en la dimensión táctica. Y viceversa. Cuando se incide sobre la dimensión técnica, esta se trabaja en contextos tácticos aunque la atención está focalizada en la dimensión técnica. Si bien la interrelación entre los aspectos tácticos y técnicos de la acción deportiva parece bien descrita y asumida a nivel académico, no resulta difícil observar en los terrenos de juego actividades donde las dimensiones tácticas y técnicas aparecen por separado y de forma descontextualizada. Especialmente cuando se trabaja con jugadores muy noveles. Pueden verse todavía, por ejemplo, tareas de pase y recepción en futbol o en baloncesto en las que no existe ni variabilidad en la ejecución del movimiento ni elementos tácticos que justifiquen las acciones de pase que se realizan.

Esta etapa 2 del modelo señala que se aprendan tanto los aspectos tácticos como los técnicos, y que ambos aprendizajes se lleven a cabo en paralelo para que se retroalimenten. Para favorecer estos aprendizajes, es preciso modular el nivel de complejidad de las dos dimensiones implicadas. Así, es necesario disminuir la dificultad de la dimensión menos importante y amplificar la participación de la dimensión sobre la que se quiere incidir. Para incidir en el aprendizaje de un aspecto de táctica individual determinado, se construyen las situaciones didácticas para que intervengan elementos técnicos significativos y relevantes aunque con un nivel de dificultad bajo o disminuido para que no interfieran negativamente en el aprendizaje del aspecto táctico. Por ejemplo, en una situación de 2 vs 1 en ataque en balonmano, para favorecer que el jugador identifique el espacio que debe atacar y si debe penetrar o pasar el balón se propone un espacio amplio, una distancia corta y que reciba el balón en carrera. Esto obliga a una toma rápida de decisiones pero evita que el jugador deba botar el balón. La misma lógica sirve cuando se pretende trabajar sobre la dimensión técnica. Se disminuye la dificultad táctica para que se aprenda o se mejore la dimensión técnica. Por ejemplo, en una 


\section{eJRIEPS 38 avril 2016}

situación de 1 vs 1 en ataque en baloncesto, se limita el uso de las manos del defensor y la distancia sobre la que puede intervenir con el objetivo de que el atacante ajuste el bote y aprenda a botar directamente con un defensor delante.

Para construir las actividades se debe tener en cuenta el nivel de dificultad sobre el aspecto concreto en el que se desea incidir, de forma que dicha actividad tenga un nivel de dificultad que esté por encima de lo que el sujeto ya sabe hacer, al mismo tiempo que se disminuye el nivel de dificultad del aspecto sobre el que no se va a incidir directamente.

Desde el punto de vista de los aprendizajes tácticos, en este nivel 2 del esquema focalizamos la atención en dos dimensiones. Por un lado en el aprendizaje de aspectos de la acción táctica individual y de toma de decisiones; y por el otro, en el aprendizaje de los medios básicos de táctica colectiva que conllevan como máximo la participación de dos atacantes y dos defensores (cruce, bloqueo, pase y va, etc.). Es decir, en situaciones de "relación de fuerzas" entre atacantes y defensores (Deleplace, 1979 ; Gréhaigne, Richard \& Griffin, 2005), pero con la participación de un número reducido de jugadores. Recuperando el ejemplo anterior, trabajamos en una situación de 2 vs 1 para incidir sobre el espacio que se debe atacar, o también en una situación de 2 vs 2 ampliando el espacio y delimitando (o no) las opciones de movimiento de uno o de los dos defensores.

Por lo que se refiere al aprendizaje de los aspectos técnicos, basta decir de acuerdo con lo señalado anteriormente que dicho aprendizaje reposa siempre sobre la eficacia de la habilidad, y aún más, considerando el entorno incierto en el que se deben ejecutar las acciones. Se prioriza el ajuste de la acción al entorno informacional en el que se debe intervenir, y a la intención perseguida en dicho entorno. Interesa, fundamentalmente, desarrollar la dimensión funcional de la habilidad de tal forma que la estructura coordinativa permita incidir con un grado de variabilidad suficiente en los diferentes contextos. En este sentido, lo que se propone responde al desarrollo de la variabilidad motriz de forma que la atención esté fijada en primer lugar en el objetivo o intención de la acción, y posteriormente en los aspectos de la estructura coordinativa.

3.- Presentación de situaciones de juego similares al deporte definitivo con aplicación de los elementos técnicos y tácticos aprendidos

En esta fase 3 empleamos situaciones de juego colectivo, juegos reducidos y juegos deportivos simplificados y modificados que mantienen semejanza estructural (y a menudo también funcional) con el deporte de referencia. En el caso de este modelo, la estructura de los juegos está pensada desde los elementos estructurales propuestos por distintos 


\section{eJRIEPS 38 avril 2016}

autores (p.e. Bayer, 1986), y también por la noción de "lógica interna" propuesta por Deleplace (1979) y después por Parlebas (1988). En este sentido, el MIT-T no se sitúa tanto bajo la influencia del TGfU como de la tradición francesa y de otros autores españoles (p.e. Lasierra \& Lavega, 1997).

El sentido inicial del uso de situaciones de juego o de juegos reducidos está en que sea un contexto colectivo (de "relación de fuerzas") de mayor complejidad estructural y funcional que en la etapa 2, vinculado estructuralmente al deporte definitivo, y por tanto con meta competitiva, que actúe como actividad o situación de referencia y que permita la aplicación de los aprendizajes realizados en la fase 2. La idea de "aplicación" no responde aquí a una perspectiva del Procesamiento de la Información y, por tanto, no comporta que en fase 2 se haya aprendido completamente el aspecto técnico o táctico y luego se aplique literalmente en situaciones de juego. Responde, más bien, a la idea de ubicar los aprendizajes que se están realizando en contextos más complejos y parecidos al deporte definitivo. Es decir, se basa en una idea de transferencia y de contextualización en niveles de aprendizaje no concluidos. Esta idea de transferencia a partir de la semejanza entre elementos estructurales (y a menudo también funcionales) facilita el ajuste de las respuestas a contextos con elementos parecidos y favorece el avance en el aprendizaje.

La participación en situaciones de juego más complejas no comporta solamente practicar los contenidos trabajados en fase 2 . Se asume y se pretende también que las situaciones de juego enriquezcan y promuevan nuevos aprendizajes a partir de generar exigencias mayores en las acciones deportivas. Para ello es necesario ajustar distintos aspectos estructurales que definen la "lógica interna" del juego. Como indican López Ros \& Castejón (2005: 45): “a) Los alumnos aplican los conocimientos adquiridos y desarrollados en la fase anterior. b) Los alumnos llevan a cabo nuevos aprendizajes como consecuencia del proceso de aplicación a las situaciones reales. c) Los profesores pueden apreciar el nivel real de aprendizaje de los alumnos y, en consecuencia, pueden planificar nuevas enseñanzas (vuelta a la fase 2) tomando como referencia lo que ha sucedido en la fase 3".

Por otro lado, el uso de los juegos reducidos debe actuar como un elemento que permita favorecer la comprensión de las acciones deportivas por parte de los aprendices. Las situaciones de juego facilitan el aprendizaje del "por qué", del "para qué", del "cuándo", del "cómo"...y en este sentido existe una coincidencia con las sugerencias derivadas del modelo inicial del TGfU. Esta facilitación deriva fundamentalmente de la funcionalidad y de la contextualización de las acciones. Es decir, aquello que los alumnos han aprendido 


\section{eJRIEPS 38 avril 2016}

en un entorno más sencillo sigue manteniendo su sentido en contextos de mayor complejidad y ello refuerza la pertinencia de estos aprendizajes. Lo mismo sucede con las nuevas acciones, no necesariamente trabajadas anteriormente, que emanan de un contexto de mayor complejidad. Ahora bien, que lo faciliten no representa que lo generen de forma automática.

Así pues, y de forma sencilla, hay dos cuestiones importantes en esta etapa. Una, que las situaciones de juego favorezcan, mediante modificaciones estructurales y funcionales, la práctica de los contenidos trabajados en la fase anterior en un contexto de mayor complejidad y dificultad, así como la aparición de nuevas acciones o de acciones más complejas. Y dos, que dichas modificaciones faciliten el desarrollo de la comprensión de los aspectos definidos en los objetivos y contenidos de aprendizaje. Es decir, que las situaciones estén alineadas con dichos objetivos y contenidos de aprendizaje.

Las situaciones de juego o los juegos modificados sirven como tarea o actividad de referencia para determinar el nivel de complejidad una vez se vuelve a las tareas de la fase 2. La evolución de las tareas técnicas y de las tareas tácticas hasta las situaciones de juego de la fase 3, y vuelta a la fase 2 es en "espiral". En este sentido las situaciones de juego o los juegos modificados sirven como actividad de referencia. Por ejemplo, en la iniciación al balonmano, el juego de "los 10 pases" puede servir como situación de referencia para trabajar la colaboración táctica en ataque y la posesión del balón sin progresión hacia portería. Ello conlleva, por ejemplo, trabajar en las tareas técnicas situaciones de pase y de recepción con oposición modificada, y en las tareas tácticas, el "pase y va" como medio básico de táctica colectiva empleando pases, recepciones y desplazamientos, y fijando la atención en el desmarque. Al mismo tiempo, la mejora producida gracias a las tareas de desarrollo de la técnica y de la táctica respectivamente, comportará enriquecer la situación de referencia y que los jugadores tengan mejor rendimiento en el mantenimiento de la posesión del balón en el juego de "los 10 pases". Evidentemente un mismo juego modificado o situación jugada puede servir como actividad de referencia para distintos contenidos técnicos y tácticos.

La secuenciación en espiral comporta emplear la misma situación de referencia modificando elementos estructurales y funcionales. Dicha secuenciación debe responder por lo menos a los criterios de: (a) aumento progresivo de la complejidad, de forma que cada vez que se vuelve a pasar por la situación de referencia ésta se muestre con un nivel superior; (b) mantenimiento de la coherencia en el desarrollo de cada serie de "espirales", es decir, las tareas y las situaciones propuestas deben responder a la misma 


\section{eJRIEPS 38 avril 2016}

intención educativa y a medida que avanza la secuencia y la complejidad dicha coherencia debe preservarse; y (c) establecimiento de continuidad, es decir, en el proceso de aprendizaje táctico y técnico no deben producirse disrupciones excesivamente grandes en el incremento de complejidad.

De acuerdo con las ideas subyacentes al aprendizaje significativo, la secuenciación en espiral comporta tomar en consideración los conocimientos previos de los jugadores y organizar y ordenar las tareas de forma que respondan al criterio de "significación lógica". Se trata, en definitiva, de establecer "una organización adecuada del contenido que facilite un aprendizaje que sea cuanto más significativo mejor, en el sentido de favorecer el establecimiento de relaciones sustantivas y no arbitrarias entre los conocimientos previos y las nuevos (...), así como la capacidad de dotar de sentido este aprendizaje" (López Ros \& Castejón, 2005: 42-43).

\section{Estrategias metodológicas}

La implementación adecuada de un modelo depende en gran medida de las estrategias didácticas y metodológicas empleadas. Puesto que el modelo es una simplificación que muestra principios y procedimientos generales, aquello que dota de realidad su aplicación es el uso de las estrategias metodológicas coherentes con el mismo. Así, por ejemplo, uno de los aspectos más analizados y discutidos del TGfU es el relativo a los principios pedagógicos del mismo (p.e. Holt, Strean \& García Bengoechea, 2002; Tan, Chow \& Davids, 2012). En nuestro caso, dividimos dichas estrategias en dos grandes ámbitos. Por un lado, las referidas al diseño y organización de las actividades; y por el otro lado, las relativas a la interacción didáctica entre el enseñante y los aprendices. Esta diferenciación de aspectos metodológicos obedece a las particularidades de los mismos, pero en realidad, en las situaciones prácticas de enseñanza, deben contemplarse como interrelacionadas. De acuerdo con la perspectiva constructivista subyacente al modelo, entendemos el proceso de enseñanza y aprendizaje como la organización de la actividad conjunta entre enseñante, aprendices y tareas bajo unas intenciones determinadas. En el centro de esta actividad conjunta se halla el triángulo didáctico o triángulo interactivo y las relaciones de interdependencia mutua de los vértices de dicho triángulo, de forma que no puede entenderse la actividad conjunta si se considera aisladamente la intervención de uno de los vértices (López Ros, 2003, 2010). 


\section{eJRIEPS 38 avril 2016}

4. 1. Estrategias básicas de diseño y organización de las tareas

De los distintos aspectos metodológicos implicados, centramos la atención en la manipulación y modificación de las condiciones de las tareas. Nos fijamos en la simplificación y la exageración en el diseño de las tareas en fase 2; y en la modificación de los elementos estructurales y funcionales en los juegos o situaciones jugadas de la fase 3.

El trabajo integrado de la técnica y de la táctica en las tareas de la fase 2 poniendo el énfasis en uno de los dos aspectos comporta emplear la exageración de aquello que se quiere evidenciar y la simplificación de los otros aspectos, minimizando o reduciendo su incidencia en la actividad. Cuando se habla, por ejemplo, de trabajar la táctica con implicación de pocos elementos técnicos hacemos hincapié en simplificar el peso relativo de la ejecución técnica o de la complejidad derivada de la participación de distintos elementos técnicos. Dicha simplificación facilita: a) que el aspecto táctico pueda trabajarse en condiciones normales o casi normales, o b) que se realce, se exagere, este aspecto táctico como consecuencia de minimizar el peso de lo técnico. Lo que resulta fundamental es que la atención del aprendiz se dirija hacia el aspecto táctico, se realice en condiciones parecidas a las del juego real, y que el nivel de dominio técnico no interfiera negativamente en el desarrollo y aprendizaje de la dimensión táctica. Lo mismo sucede si lo que se pretende es incidir sobre la dimensión técnica. De lo que se trata es que la complejidad del problema táctico no interfiera negativamente en el aprendizaje y/o en la mejora de la eficacia del movimiento. Como se indicaba en su momento: "Si las tareas tienen que contener los aspectos técnicos y tácticos, la incidencia educativa en uno u otro se hará a partir de simplificar uno de los componentes (técnico o táctico) para que "resalte" el dominio objeto de mejora" (López Ros \& Castejón, 2005: 44). Ello comporta que muy a menudo la simplificación y la exageración actúan como un binomio interrelacionado, de forma que cuando se simplifica la implicación de un aspecto ello conlleva la exageración de otro.

El uso de estas estrategias es coincidente con las aportaciones de otros modelos. Así, por ejemplo, el TGfU establece la exageración como uno de sus principios pedagógicos fundamentales, y también sugiere emplear la simplificación como recurso. Igualmente, distintos trabajos que abordan los aspectos comunes entre el TGfU y el "Constraint-led approach" o la "pedagogía no lineal" señalan estos principios pedagógicos como ámbitos de coincidencia (Clemente, 2012; Renshaw, Chow, Davids \& Hammond, 2010; Tan, Chow \& Davids, 2012). Una de las aportaciones probablemente más destacadas de la 


\section{eJRIEPS 38 avril 2016}

"Constraint-led approach" para la enseñanza deportiva sea que obtengamos una mayor comprensión acerca de la manipulación de los constreñimientos para favorecer la aparición o emergencia de determinadas acciones. Especialmente, los constreñimientos relativos a las tareas.

El diseño ajustado de situaciones de juego o de juegos modificados en fase 3 comporta la manipulación de distintos aspectos estructurales y/o funcionales con el objetivo de ajustar el nivel de complejidad de los juegos. En el momento en que se desarrolló el MIT-T las estrategias de intervención se pensaban desde una mirada estructural-funcional de los deportes (p.e. Bayer, 1986). Ésta era una mirada que focalizaba la atención en los elementos presentes desde un punto de vista "estático". De ahí que se proponga modificar aspectos como el espacio de juego, el número de jugadores, las posibilidades de acción de los jugadores, entre otros aspectos. Por ejemplo, para trabajar el mantenimiento de la posesión del balón pueden emplearse situaciones de desigualdad numérica, o de igualdad numérica con el campo más grande, o puede incorporarse un "jugador comodín" que siempre interviene con el equipo que tiene la posesión del balón. En el momento de la aparición del MIT-T, y a pesar de que las teorías sistémicas tenían un cierto auge, no existía todavía un análisis profundo desarrollado desde la teoría de la complejidad ni de la dimensión dinámica. En la actualidad existe un fuerte empuje en el estudio de la teoría de la complejidad aplicada al análisis de los deportes (p.e. Lago, 2010; Martín \& Lago, 2005), y también en la investigación sobre los "small-sided games", sus características, el impacto de la modificación de determinados elementos, y sus efectos en las acciones de los jugadores (p.e. Diaz-Cidoncha et al., 2014; Silva et al., 2014).

4. 2. Estrategias básicas de interacción didáctica

De acuerdo con lo señalado hasta el momento, la perspectiva bajo la que se considera la interacción didáctica en el MIT-T tiene que ver con el proceso de scaffolding y con el ajuste de la ayuda pedagógica para que esta sea contingente. Lo que resulta fundamental es que el profesor vaya ajustando sus intervenciones de forma que permita ir avanzando a los alumnos en el aprendizaje (Coll, Onrubia \& Mauri, 2008; López Ros, 2003). Parece, en este orden de cosas, inadecuado ofrecer determinados recursos semióticos y de intervención didáctica de manera que su uso se pueda generalizar de forma prescriptiva. La actividad conjunta se construye en cierta medida sobre la marcha, y resulta imposible anticipar completamente la evolución de los procesos interactivos ni definir a priori qué tipo preciso de recurso se empleará y en qué momento. Algunos estudios realizados en 


\section{eJRIEPS 38 avril 2016}

condiciones reales de enseñanza y aprendizaje deportivo muestran dichos aspectos dinámicos e imprevisibles de la organización de la actividad conjunta (p.e. López-Ros, 2013; López-Ros, Llobet \& Comino, 2013).

Ahora bien, a pesar de que el proceso interactivo es parcialmente imprescindible, no es menos cierto que responde a criterios de planificación docente en los que se establecen, por lo menos, los objetivos y las directrices básicas de intervención metodológica que acaban configurando el escenario interactivo. En este contexto, consideramos distintos tipos de recursos semióticos por parte del enseñante. Por un lado, el uso de recursos icónicos; y por otro lado, el uso de recursos lingüísticos. El uso de recursos icónicos comporta emplear las imágenes, y por tanto la observación, como herramienta de aprendizaje comprensivo (p.e. Gréhaigne, Caty \& Godbout, 2010). Se pueden usar herramientas tecnológicas o también imágenes antes, después o durante las mismas situaciones prácticas de enseñanza, por ejemplo, mediante situaciones de juego real “congeladas" (el profesor para el juego o situación de forma que todos los participantes se quedan quietos donde estaban y se organiza la reflexión a partir de esta imagen).

El uso de los recursos lingüísticos para favorecer un aprendizaje comprensivo está ampliamente estudiado y desarrollado. Uno de los recursos propuestos con mayor profusión desde los distintos modelos de enseñanza comprensiva son las preguntas (p.e. Gréhaigne, Caty \& Godbout, 2010; López Ros, 2003; Wright \& Forrest, 2007), puesto que permiten y facilitan el desarrollo del pensamiento crítico (Gréhaigne, Richard \& Griffin, 2005) y de la conciencia táctica (Mitchell, Oslin \& Griffin, 2006) y tienen un papel fundamental en el desarrollo de la comprensión. Algunos de estos trabajos sugieren la necesidad de planificar también el tipo de preguntas previamente a la intervención debido a que se considera un aspecto clave en el desarrollo de la conciencia táctica (Mitchell, Oslin \& Griffin, 2006).

En el momento en el que se planteó el MIT-T no se proponían estrategias interactivas específicas, aludiéndose de forma global a la necesidad de facilitar la comprensión, de establecer un sistema de significados compartidos que permitieran el desarrollo de un aprendizaje más significativo, y de ajustar la ayuda pedagógica. Sin embargo, el hecho de situar el modelo en esta perspectiva de la actividad conjunta nos llevó a considerar los procesos interactivos y el uso de recursos semióticos guiados por autores como Edwards y Mercer (1987), Mercer (1995) o Lemke (1993), que muestran una visión más pragmática que formal de los recursos lingüísticos. Tenía interés considerar, por ejemplo, el uso de las preguntas no tanto desde su aspecto formal sino en función de la intención 


\section{eJRIEPS 38 avril 2016}

perseguida: no es lo mismo una pregunta que persigue obtener una respuesta desde el desconocimiento del interlocutor, que una pregunta que tiene una función de demanda (petición) de acción, o una pregunta que busca indagar si el interrogado sabe la respuesta correcta (a modo de descubrimiento guiado). Desde este punto de vista cabe señalar la pertinencia de cualquier recurso lingüístico (preguntas, prescripciones, etiquetados, etc.) siempre que su orientación permita el establecimiento y enriquecimiento de un sistema de significados compartidos, contextualizados, y el desarrollo de la comprensión.

Asimismo, el uso de determinados recursos lingüísticos cabe considerarlos según la función instruccional del segmento interactivo en el que se hallan inmersos los sujetos. Un segmento interactivo puede entenderse como la unidad básica de organización de la actividad conjunta (puede verse una revisión del estado de la cuestión en Coll, Onrubia \& Mauri, 2008). Así, el enseñante y los aprendices pueden estar, por ejemplo, en un segmento de organización de la actividad en el cuál el profesor lleva a cabo una recapitulación de lo que se hizo el día anterior antes de plantear la actividad; o en un segmento de discusión tras una tarea practicada, o en un segmento de práctica guiada en la que el profesor interviene esporádicamente para guiar las acciones de los jugadores. En cada segmento interactivo, y de acuerdo con la función instruccional del mismo, el sentido de las preguntas, del etiquetado o de cualquier otra forma de intervención lingüística puede variar sustancialmente. Dicho esto, es cierto que el uso de recursos que permitan la contextualización (por ejemplo recapitulaciones o recapitulaciones anticipatorias), que faciliten el establecimiento y construcción de nuevos significados anclados a partir de conocimientos previos (por ejemplo etiquetados), que faciliten la conexión entre la acción y el conocimiento de carácter más abstracto (por ejemplo, el uso de situaciones congeladas conectando lo que sucede en la práctica con conceptos tácticos colectivos o con aspectos de los principios estratégicos), y que permitan indagar nuevas respuestas, facilitará el desarrollo de la comprensión y de un aprendizaje más significativo. Desde este punto de vista, algunos trabajos han mostrado el uso de los recursos semióticos y su función según el segmento interactivo en el que se emplean (López-Ros, 2003; López-Ros, Llobet \& Comino, 2013). En estos casos se evidencia también que el uso de preguntas es un elemento fundamental para facilitar un aprendizaje comprensivo.

Al igual que otros modelos, entendemos que el uso de los recursos semióticos juntamente con el nivel de dificultad de la situación planteada son dos pilares fundamentales para garantizar el desarrollo de la comprensión. 


\section{eJRIEPS 38 avril 2016}

\section{Conclusión}

En el momento de la aparición del MIT-T existía, como ya hemos indicado, un fuerte debate acerca de si era mejor empezar la enseñanza por la técnica, por la táctica o por ambos aspectos. Distintos trabajos, inclusive aquellos que tenían una intervención didáctica de larga duración (p.e. García Herrero \& Ruiz Pérez, 2003), no mostraban conclusiones definitivas al respecto. En la actualidad estamos en un momento en el que debe profundizarse la comprensión de cómo interaccionan el dominio técnico y táctico, y si bien disponemos de múltiples trabajos de la mejora de la acción táctica y del predominio de lo táctico sobre lo técnico, debemos avanzar en el conocimiento del desarrollo compensado entre ambas dimensiones.

Recientemente también se están discutiendo las aportaciones realizadas desde los modelos de corte más táctico (Oslin \& Mitchell, 2006; Harvey \& Jarrett, 2013; Stolz \& Pill, 2014), y se está señalando la necesidad de disponer de evidencias empíricas que soporten los supuestos teóricos de estos modelos de claro predominio táctico, y en especial que se facilite el uso de estos modelos en la enseñanza en los centros educativos (Stolz \& Pill, 2014). El MIT-T es un modelo teórico, que si bien ha tenido un cierto nivel de impacto (Barrachina, 2012; Sánchez-Gómez, Devís-Devís \& NavarroAdelantado, 2014), necesita soporte empírico que evidencie su pertinencia y funcionalidad. Algunas investigaciones han empleado este modelo para distintos tipos de estudios. Disponemos de trabajos que comparan la eficacia de distintos enfoques empleando el MIT-T en el grupo técnico-táctico (Castejón et al., 2002), trabajos que analizan el proceso de cesión y de traspaso de la responsabilidad en el aprendizaje empleando la enseñanza integrada técnico-táctica en la unidad didáctica (López-Ros, 2013; López-Ros \& Llobet, 2013), estudios de comparación de las bases teóricas de distintos modelos incluyendo el MIT-T (Barrachina, 2012; Contreras, García \& Gutiérrez, 2001), y también el uso del MIT-T y de otros modelos de enseñanza comprensiva para diseñar propuestas metodológicas en otros deportes, como por ejemplo en la iniciación a los deportes de lucha (Avelar Rosa \& Figueiredo, 2009).

En este contexto es necesario mejorar y avanzar en distintos ámbitos, tanto desde el punto de vista del desarrollo teórico como de la obtención de evidencias empíricas. Algunos aspectos especialmente relevantes son, por un lado, disponer de una mejor comprensión del funcionamiento de los procesos metodológicos de simplificación y exageración. Como hemos indicado en otro apartado, las aportaciones surgidas de la “Constraint-led approach" (Clemente, 2012; Renshaw, Chow, Davids \& Hammond, 2010; 


\section{eJRIEPS 38 avril 2016}

Tan, Chow \& Davids, 2012) pueden ofrecernos respuestas interesantes, aunque queda por ver la compatibilidad de dichas aportaciones desarrolladas bajo una perspectiva teórica y epistemológica diferentes a la enseñanza comprensiva. En este mismo orden de cosas se ubican los avances que podamos obtener de la investigación con los "smallsided games". Debemos profundizar en la coherencia entre estas aportaciones y el marco teórico de referencia en el que se sitúa el MIT-T. Las aportaciones de perspectivas teóricas basadas en parte en postulados ecológicos y, por otra parte en postulados constructivistas, como el "Situated learning", podrían ser un buen punto de encuentro (Rovegno, 2006).

Asimismo, debemos avanzar en la comprensión de los procesos de secuenciación de las tareas, y en la relación entre las tareas de menor complejidad estructural (tareas de fase 2) y las situaciones de juego (tareas de fase 3). Disponemos de evidencias que señalan que el uso de tareas de referencia y de modificaciones sobre las mismas permiten facilitar el proceso de cesión y de traspaso (López Ros, 2013), sin embargo queda todavía mucho camino por recorrer. Lo mismo sucede en lo relativo al uso de recursos semióticos en el contexto de los procesos de scaffolding. Al igual que señalábamos anteriormente, el avance en el conocimiento de los procesos interactivos debe realizarse de forma coherente con la perspectiva teórica el modelo si lo que se desea es favorecer un aprendizaje comprensivo.

Desde el punto de vista de los contenidos de enseñanza, resulta necesario profundizar en las relaciones entre los dominios técnico y táctico de las acciones, y también en el desarrollo del pensamiento táctico o de la llamada inteligencia táctica. Este segundo aspecto es el eje nuclear que conecta las acciones individuales en el seno del desarrollo colectivo. Existen en la actualidad distintos trabajos que abordan las coordinaciones entre los jugadores y el proceso de emergencia de dichas coordinaciones, de la misma manera que hay trabajos que muestran el papel del pensamiento y de la conciencia táctica dentro del juego colectivo. En el caso del MIT-T, el desarrollo del pensamiento táctico y de la comprensión es fundamental y debemos profundizar en las estrategias de aprendizaje y mejora de dicha conciencia táctica. Por el momento, entendemos que los modelos de enseñanza comprensiva pueden ser un buen entorno en el que conectar las dimensiones micro (acción táctica y técnica individual) y macro (principios estratégicos y táctica colectiva) de las acciones deportivas.

Más allá de considerar aspectos globales del MIT-T, no debe olvidarse que dicho modelo debe concretarse y ajustarse a los distintos tipos o familias de deportes. Es decir, y con el 


\section{eJRIEPS 38 avril 2016}

objetivo de facilitar su uso en el quehacer diario de los enseñantes, deberíamos avanzar en el desarrollo específico del modelo en función de diferentes familias o grupos de deportes. Un modelo tiene sentido, en última instancia, si es útil y funcional para los enseñantes y aprendices.

\section{Bibliografía}

Ausubel, D.P. (2000). The acquisition and retention of knowledge. Dordrecht : Kluwer Academic Publishers.

Ausubel, D.P., Novak, J.D., \& Hanesian, H. (1990) (2 $2^{\mathrm{a}}$ ed.). Psicología educativa. Un punto de vista cognoscitivo. México D.F. : Trillas.

Avelar Rosa, B. \& Figueiredo, A. (2009). La iniciación a los deportes de combate: Interpretación del fenómeno lúdico luctatorio. Revista de Artes Marciales Asiáticas, 4(3), 44-57.

Barrachina, J. (2012). ¿Cómo lo estamos haciendo? Revisión crítica de algunos modelos de iniciación deportiva. In E. Sebastiani, D. Blázquez (Coords.), ¿Cómo formar un buen deportista? Un modelo basado en competencias, (pp. 87-111). Barcelona : Inde.

Bayer, C. (1986). La enseñanza de los juegos deportivos. Barcelona : Hispano Europea.

Bayer, C. (1987). Técnica del balonmano. Barcelona : Hispano Europea.

Blázquez, D. (1986). Iniciación a los deportes de equipo. Barcelona : Martínez Roca.

Bonnet, J.P. (1988). (2 $2^{a}$ ed.) Vers une pédagogie de l'acte moteur. Réflexions critiques sur les pédagogies sportives. Paris : Vigot.

Bouthier, D. (1984). Sports collectifs: contribution à l'analyse de l'activité et éléments pour une formation tactique essentielle. L'exemple du Rugby. Paris : INSEP

Bouthier, D. (2014). Iniciación y perfeccionamiento en los deportes colectivos: desarrollo de la pertinencia de la toma de decisiones en el juego en relación con otros elementos de la acción. In V. López-Ros, J. Sargatal (Eds.), El aprendizaje de la acción táctica, (pp. 99-133). Girona : UdGUniversitat de Girona.

Bunker, D. J., \& Thorpe, R. D. (1982). A model for the teaching of games in secondary school. Bulletin of Physical Education, 18(1), 5-8.

Castejón, F.J. (1995). Fundamentos de iniciación deportiva y actividades físicas organizadas. Madrid : Dykinson. 


\section{eJRIEPS 38 avril 2016}

Castejón, F.J. (2003) (Coord.). Iniciación deportiva: la enseñanza y el aprendizaje comprensivo en el deporte. Sevilla : Wanceulen.

Castejón, F.J. (2010) (Coord.). Deporte y enseñanza comprensiva. Sevilla : Wanceulen.

Castejón, F.J., \& López Ros, V. (1997). Iniciación deportiva. In F.J. Castejón (Coord.), Manual del Maestro Especialista en Educación Física, (pp. 137-172). Madrid : Pila Teleña.

Castejón, F.J., Giménez Fuentes-Guerra, F.J., Jiménez Jiménez, F., \& López Ros, V. (2003). Concepción de la enseñanza comprensiva del deporte: modelos, tendencias y propuestas. In F.J. Castejón (Coord.), Iniciación deportiva: la enseñanza y el aprendizaje comprensivo en el deporte, (pp. 17-34). Sevilla : Wanceulen.

Castejón, F.J. et al. (2002). La enseñanza del deporte con diferentes estrategias de enseñanza: técnica, táctica y técnico-táctica. Revista de Educación Física. Renovar la teoría y la práctica, 86, 27-33.

Chen, W., Rovegno, I., Cone, S., \& Cone, T. (2012). An accomplished teacher's use of scaffolding during a second-grade unit on designing games. Research Quarterly for Exercise and Sport, 83(2), 221-234.

Clemente, F. (2012). Princípios pedagógicos dos Teaching Games for Understanding e da Pedagogia Nao-Linear no ensino da Educaçao Física. Movimento, 18(2), 315-335.

Coll, C. (1988). Significado y sentido en el aprendizaje escolar. Reflexiones en torno al concepto de aprendizaje significativo. Infancia y Aprendizaje, 41, 131-142.

Coll, C., Onrubia, J., \& Mauri, T. (2008). Supporting learning in educational contexts: the exercise of educational influence and the analysis of teaching. Revista de Educación, 346, 33-70.

Contreras, O., García, L.M., \& Gutiérrez, D. (2001). Análisis crítico a la metodología comprensiva para la enseñanza de los deportes. Actas del XIX Congreso Nacional de Educación Física, Vol. II, (pp. 745-759). Murcia : Universidad de Murcia.

Cubero, R. (2005). Perspectivas constructivistas. La intersección entre el significado, la interacción y el discurso. Barcelona : Graó.

Daniels, H. (2001). Vygotsky and Pedagogy. Londres : Routledge Falmer.

Deleplace, R. (1979). Rugby de movement - Rugby total. Paris : Éducation Physique et Sports.

Devís, J. (1990). Renovación pedagógica de la Educación Física: hacia dos alternativas 


\section{eJRIEPS 38 avril 2016}

de acción I. Perspectivas de la Actividad Física y el Deporte, 4, 5-7.

Devís, J. (1992) Bases para una propuesta de cambio en la enseñanza de los juegos deportivos. In J. Devís, C. Peiró, Nuevas perspectivas curriculares en educación física: la salud y los juegos modificados, (pp. 141-159). Barcelona : Inde.

Devís, J. \& Peiró, C. (1992). Orientaciones para el desarrollo de una propuesta de cambio en la enseñanza de los juegos deportivos. In J. Devís, C. Peiró, Nuevas perspectivas curriculares en educación física: la salud y los juegos modificados, (pp. 161-184). Barcelona: Inde.

Díaz-Cidoncha, J. et al. (2014). Quantification and analysis of offensive situations in different formats of sided games in soccer. Journal of Human Kinetics, 44, 129137.

Díaz-Cueto, M., Hernández-Álvarez, J.L. \& Castejón, F.J. (2010). Teaching Games for Understanding to in-service physical education teachers: Rewards and barriers regarding the changing model of teaching sport. Journal of Teaching in Physical Education, 29, 378-398.

Edwards, D. \& Mercer, N. (1987). Common knowledge. The development of understanding in the classroom. Londres : Methuen \& co.

Famose, J-P. (1991). Apprentissage moteur et résolution de problèmes. In J-P. Famose, Ph. Fleurance, Y. Touchard (Dirs.), L'apprentissage moteur. Rôle des représentations, (pp. 23-57). Paris : EPS.

García Herrero, J.A. \& Ruiz Pérez, L.M. (2003). Análisis comparativo de dos modelos de intervención en el aprendizaje del balonmano. Revista de Psicología del Deporte, 12(1), 55-66.

Gréhaigne, J.F., Caty, D., \& Godbout, P. (2010). Modelling ball circulation in invasion team sports: a way to promote learning games through understanding. Physical Education and Sport Pedagogy, 15(3), 257-270.

Gréhaigne, J.F., Godbout, P., \& Bouthier, D. (1999). The foundations of tactics and strategy in team sports. Journal of Teaching in Physical Education, 18, 159-174.

Gréhaigne, J.F., Richard, J.F., \& Griffin, L. (2005). Teaching and learning team sports and games. New York : Routledge.

Gréhaigne, J. F., Wallian, N., \& Godbout, P. (2005). Tactical-decision learning model and students' practices. Physical Education and Sport Pedagogy, 10(3), 255-269.

Griffin, J. \& Patton, K. (2005). Two decades of Teaching Games for Understanding: looking at the past, present and future. In L. Griffin, J. Butler (2005) (Eds.), 


\section{eJRIEPS 38 avril 2016}

Teaching Games for Understanding: Theory, research and practice, (pp. 1-17). Champaign : Human Kinetics.

Griffin, L. \& Butler, J. (2005) (Eds.). Teaching Games for Understanding: Theory, research and practice. Champaign : Human Kinetics.

Griffin, L., Brooker, R \& Patton, K. (2005). Working towards legitimacy: two decades of Teaching Games for Understanding. Physical Education and Sport Pedagogy, 10, 213-223.

Harvey, S. y Jarrett, K. (2013). A review on the game-centred approaches to teaching and coaching literature since 2006. Physical Education and Sport Pedagogy, 19(3), 278-300.

Holt, N. L., Strean, W.B. \& García Bengoechea, E. (2002). Expanding the Teaching Games for Understanding Model: New avenues for future research and practice. Journal of Teaching in Physical Education, 21(4), 162-176.

Kirk, D. \& MacPhail, A. (2002). Teaching Games for Understanding and Situated Learning: Rethinking the Bunker-Thorpe Model. Journal of Teaching in Physical Education, 21(4), 177-179.

Lago, C. (2010) La toma de decisiones desde la perspectiva de los sistemas complejos. La influencia de las variables contextuales de la competición en el comportamiento de los jugadores en los deportes de equipo. In V. López Ros, J. Sargatal (Eds.), La táctica deportiva y la toma de decisiones. Girona : UdGUniversitat de Girona.

Lasierra, G. \& Lavega, P. (1997) (2ª ed.). 1015 juegos y formas jugadas de iniciación a los deportes colectivos. Barcelona: Paidotribo.

Lemke, J. (1993). Talking science: language, learning and values. Norwood : Ablex Publishing Corporation.

López Ros, V. (2003). Enseñanza, aprendizaje e iniciación deportiva: la interacción educativa en el aprendizaje comprensivo del deporte. In F.J. Castejón (Coord.), Iniciación deportiva: la enseñanza y el aprendizaje comprensivo en el deporte, (pp. 111-140). Sevilla : Wanceulen.

López Ros, V. (2010). Perspectiva constructivista del aprendizaje y de la enseñanza del deporte. In F.J. Castejón (Coord.), Deporte y enseñanza comprensiva, (pp. 3561). Sevilla : Wanceulen.

López Ros, V. (2013). La organización de la actividad conjunta en la enseñanza escolar de los deportes colectivos. In F.J. Castejón, F.J. Giménez Fuentes-Guerra, F. 


\section{eJRIEPS 38 avril 2016}

Jiménez Jiménez, V. López Ros (Coords.), Investigaciones en Formación Deportiva, (pp. 41-64). Sevilla : Wanceulen.

López Ros, V. \& Castejón, F.J. (1998a). Técnica, táctica individual y táctica colectiva. Teoría de la implicación en el aprendizaje y la enseñanza deportiva (I). Revista de Educación Física. Renovar la teoría y la práctica, 68, 5-9.

López Ros, V. \& Castejón, F.J. (1998b). Técnica, táctica individual y táctica colectiva. Implicación en el aprendizaje y la enseñanza deportiva (práctica). Revista de Educación Física. Renovar la teoría y la práctica, 68, 12-16.

López Ros, V. \& Castejón, F.J. (2005). La enseñanza integrada técnico-táctica de los deportes en edad escolar. Apunts. Educació Física i Esports, 79, 40-48.

López-Ros, V. \& Llobet, B. (2013). The joint activity in a teaching and learning sequence of rugby. A descriptive analysis. In N. Balagué et al. (Eds.), Book of abstracts. $18^{\text {th }}$ Annual Congress of the European College of Sport Sciences, (p. 433). Colonia : ECSS, European College of Sports Sciences.

López-Ros, V., Llobet, B. \& Comino, J. (2013). Patterns of joint activity and semiotic devices in teaching sport. A case study. In B. Carnel, J. Moniotte (Eds.), Intervention, Recherche et Formation: Quels enjeux, quelles transformations?, (pp. 296-309). Amiens : Université de Picardie Jules Verne.

López-Ros, V., Castejón, F.J., Bouthier, D. \& Llobet, B. (2015). Modelos para una enseñanza comprensiva del deporte. Espacios comunes para el encuentro (y algún desencuentro). Ágora para la Educación Física y el Deporte, 17(1), 45-60.

Mahlo, F. (1974). La acción táctica en el juego. La Habana: Pueblo y Educación.

Martín, R. \& Lago, C. (2005). Deportes de equipo. Comprender la complejidad para elevar el rendimiento. Barcelona : Inde.

Mercer, N. (1995). The guided construction of knowledge. Talk among teachers and learners. Clevedon : Multilingual Matters.

Oslin, J. \& Mitchell, S. (2006). Game-centered approaches to teaching physical education. In D. Kirk, D. MacDonald, M. O'Sullivan (Eds.), The Handbook of Physical Education, (pp. 627-651). London : Sage.

Parlebas, P. (1988). Elementos de sociología del deporte. Málaga : Universidad Internacional Deportiva/ Junta de Andalucía.

Rensahw, I., Chow, J.Y., Davids, K. \& Hammond, J. (2010). A constraints-led perspective to understanding skill acquisition and game play: a basis for integration of motor 


\section{eJRIEPS 38 avril 2016}

learning theory and physical education praxis? Physical Education and Sport Pedagogy, 15(2), 117-137.

Rink, J. (1996) (Ed.). Tactical and skill approaches to teaching sport and games. Journal of Teaching in Physical Education, 4(15) (Volumen monográfico).

Rink, J. (2001). Investigating the assumptions of pedagogy. Journal of Teaching in Physical Education, 20, 112-128.

Rivière, A. (1988). La psicología de Vygotski. Madrid : Visor.

Rovegno, I. (2006). Situated perspectives on learning. In D. Kirk, D. MacDonald, M. O'Sullivan (Eds.), The Handbook of Physical Education, (pp. 262-274). London : Sage.

Rovegno, I. \& Dolly, J.P. (2006). Constructivist perspectives on learning. In D. Kirk, D. MacDonald, M. O'Sullivan (Eds.), The Handbook of Physical Education, (pp. 242261). London : Sage.

Ruiz Pérez, L.M. (1995). Competencia motriz. Elementos para comprender el aprendizaje motor en educación física escolar. Madrid : Gymnos.

Ruiz Pérez, L.M. (2001). Conocimiento sobre las acciones y competencia motriz. In L. M. Ruiz Pérez (Coord.), Desarrollo, comportamiento motor y deporte, (pp. 165-186). Madrid : Síntesis.

Sánchez-Sánchez, R., Devís-Devís, J. \& Navarro-Adelantado, V. (2014). El modelo Teaching Games for Understanding en el contexto internacional y español: una perspectiva histórica. Ágora para la Educación Física y el Deporte, 16(3), $197-$ 213.

Silva, P. et al. (2014). Field dimension and skill level constrain team tactical behaviours in small-sided and conditioned games in football. Journal of Sports Sciences, 32(20), 1888-1896.

Stolz, S. \& Pill, S. (2014). Teaching games and sport for understanding: exploring and reconsidering its relevance in physical education. European Physical Education Review, 20(1), 36-71.

Tan, C.W.K., Chow, J.Y. \& Davids, K. (2012). "How does TGfU work?": Examining the relationship between learning design in TGfU and a nonlinear pedagogy. Physical Education and Sport Pedagogy, 17(4), 331-348.

Teodorescu, L. (1977). Théorie et méthodologie des jeux sportifs. Paris : Vigot. 


\section{eJRIEPS 38 avril 2016}

Turner, A.P. \& Martinek, T.J. (1992). A comparative analysis of two models for teaching games: Technique approach and Game-Centered (Tactical focus) Approach. International Journal of Physical Education, 29(4), 15-31.

Van de Pol, J., Volman, M. \& Beishuizen, J. (2010). Scaffolding in teacher-student interaction: A decade of research. Educational Psychological Review, 22, 271-296.

Van de Pol, J., Volman, M. \& Beishuizen, J. (2011). Patterns of contingent teaching in teacher-student interaction. Learning and Instruction, 21, 46-57.

Vigotsky, L.S. (1979). El desarrollo de los procesos psicológicos superiores. Barcelona : Crítica.

Vila, I. (1987). Vigotski: la mediació semiótica de la ment. Vic : Eumo.

Wood, D., Bruner, J. S., \& Ross, G. (1976). The role of tutoring in problem solving. Journal of child psychology and psychiatry, 17(2), 89-100.

Wright, J. \& Forrest, G. (2007). A social semiotic analysis of knowledge construction and games centred approaches to teaching. Physical Education and Sport Pedagogy, 12(3), 273-287.

Wright, S., McNeill, M. \& Fry, J.M. (2009). The tactical approach to teaching games from teaching, learning and mentoring perspectives. Sport, Education and Society, 14(2), 223-244. 